\title{
STUDY OF ATMOSPHERIC AEROSOLS AND MIXING LAYER BY LIDAR
}

\author{
Federico Angelini*, Francesca Barnaba, Tony Christian Landi, Luca Caporaso and Gian Paolo Gobbi \\ ISAC-CNR, Via del Fosso del Cavaliere 100, 00133 Rome, Italy
}

\begin{abstract}
The LIDAR (laser radar) is an active remote sensing technique, which allows for the altitude-resolved observation of several atmospheric constituents. A typical application is the measurement of the vertically resolved aerosol optical properties. By using aerosol particles as a marker, continuous determination of the mixing layer height (MLH) can also be obtained by LIDAR. Some examples of aerosol extinction coefficient profiles and MLH extracted from a 1-year LIDAR data set collected in Milan (Italy) are discussed and validated against in situ data (from a balloon-borne optical particle counter). Finally a comparison of the observation-based MLH with relevant numerical simulations (mesoscale model MM5) is provided.
\end{abstract}

\section{INTRODUCTION}

The LIDAR (LIght Detection And Ranging) is an instrument conceptually similar to the radar, but using visible, infrared or ultraviolet laser light instead of microwaves. Due to the shorter radiation wavelength, it allows remote sensing of small bodies, typically of the order of microns, such as atmospheric aerosols (or particulate matter, PM). The quantitative observation of the atmospheric PM is important in many fields, in particular in climate and air quality studies.

The globally averaged aerosol net effect on climate is a cooling of the Earth surface. The last IPCC report $^{(1)}$ estimates a mean direct radiative forcing between -0.9 and $-0.1 \mathrm{~W} \mathrm{~m}^{-2}$, and an indirect radiative forcing, due to the alteration of cloud properties, between -1.8 and $-0.3 \mathrm{~W} \mathrm{~m}^{-2}$. It must be noted, however, that the uncertainties associated with these values are rather high, especially if compared with those associated with the gaseous components. As a consequence, aerosol effects represent a major uncertainty in the evaluation of the human contribution to the radiative balance alteration.

With regard to air quality, it is now evident that inhalation of PM may cause health damages, so that national and international regulations are becoming more and more restrictive in terms of PM mass concentration threshold levels. The aerosol observation/ monitoring then plays a key role also in air quality studies, and the possibility of remotely sense PM levels, either from space or ground, is a current research task ${ }^{(2)}$. In fact, specific initiatives, such as IDEA (www.star.nesdis.noaa.gov/smcd/spb/aq/), funded by the US NASA-EPA-NOAA, or the Italian project QUITSAT (www.quitsat.it), funded

\footnotetext{
* Corresponding author: f.angelini@isac.cnr.it
}

by the national space agency (ASI), aim at the improvement of air quality assessment by exploiting the huge information provided by space-based sensors.

In this framework, the LIDAR capabilities of profiling the aerosol optical properties represent a unique opportunity to relate the total aerosol amounts observed from space to the relevant fraction measured at the ground. Other important opportunities offered by LIDARs in air quality studies are: (1) the monitoring of the mixing layer height (MLH), useful for pollutants dispersion evaluation $^{(3)}$, and (4) estimating the contribution of some 'natural' aerosols (as desert dust) to total aerosol loads, and thus to PM10 levels ${ }^{(4)}$.

This study briefly describes the LIDAR technique and, based on LIDAR data collected in Milan (Italy), presents some methodologies to investigate the aerosol vertical distribution and MLH daily evolution. Some validation of the LIDAR data through a comparison with aerosol 'in situ' data is also provided. Finally a comparison of the observation-based MLH with numerical simulations by a mesoscale model is also given.

\section{METHODS}

The LIDAR technique employs light emitted by a laser source to sound the atmosphere with rather high vertical resolution. Optical telescopes and photomultipliers (or photo-diodes) are used to collect and detect the light backscattered by the atmosphere. A thorough description of this technique can be found for example in Measures ${ }^{(5)}$. Simple (single-wavelength) and easily deployable LIDAR systems can be used to derive vertical profiles of the atmospheric aerosol optical properties, as the aerosol extinction coefficient (at the laser wavelength). An accurate retrieval of aerosol extinction is 


\section{F. ANGELINI ET AL.}

however not straightforward. In fact, in nearly all the existing LIDARs (including Raman ones), some assumptions are needed to solve the LIDAR equation. This is mainly due to the fact that the aerosol extinction cross section is related to the backscatter aerosol cross section through a phase function, which depends on the (unknown) aerosol microphysical properties (size distribution, shape and refractive index). To minimise the impact of these assumptions, several inversion schemes have been developed ${ }^{(6-8)}$. In these applications, operatorbased data retrievals are generally used as visual data inspection is often necessary.

Conversely, automated algorithms can be used to estimate the MLH by LIDAR, so that, for this purpose, unattended operations are possible. The idea behind the MLH identification through aerosols data is that these represent a good marker for the mixed layer, i.e. particles mixing due to turbulent motions of the lower troposphere is fast enough to allow MLH identification through investigation of the aerosol load gradient. Most of the available procedures used to retrieve the MLH from LIDAR data can be classified as the following:

(1) Threshold methods: It is based on the search of the height at which the signal falls under a fixed threshold $^{(9)}$.

(2) Gradient methods: It is based on the determination of the minima of the logarithmic rangecorrected signal (RCS) gradient ${ }^{(10,11)}$.

(3) Variance methods: It is based on the determination of the maxima in the temporal variance of the signal ${ }^{(12)}$.

It should be noted here that all these LIDAR retrievals can be performed only below cloud or fog levels. In fact, in the presence of rain, clouds or fog the decrease in signal is mainly due to the extinction caused by these hydrometeors rather than to a reduction in the aerosol cross section. Therefore, the essential requirement for the LIDAR-based MLH detection fails. The algorithm implemented in the MLH analysis here belongs to the gradient method category and will be briefly described in the following section.

Selected examples of both aerosol extinction retrievals and MLH estimation will be provided based on the data collected using an automated LIDAR ceilometer (Vaisala LD-40) in the framework of the QUITSAT project. The ceilometer, operating at $855 \mathrm{~nm}$, ran in the Milan urban area (Torre Sarca) for over 200 days in the period January 2007 to February 2008. Aerosol backscatter profiles were acquired every $15 \mathrm{~s}$ and averaged over $15 \mathrm{~min}$ to achieve a better signal-to-noise ratio. So, 96 profiles per day are available. Due to the instrumental limitations, the lowest height the LIDAR can observe is $\sim 60 \mathrm{~m}$. Additional information about LIDAR ceilometers can be found in Eresmaa et al. ${ }^{(13)}$ and references therein.

On specific dates, a balloon-borne (BB) optical particle counter (OPC, GRIMM 1.108 'Dustcheck') was also operated by the University of Milan 'Bicocca', collecting aerosol size distribution data between 0.3 and $20 \mu \mathrm{m}$ up to about $600 \mathrm{~m}$ agl.

For some case studies, numerical simulations of MLH time evolution were also performed by the PSU/NCAR mesoscale model MM5 (V3-6) employing the non-local MRF boundary layer parameterisation $^{(14)}$ and a high-resolution land use $(1 \times 1 \mathrm{~km})$.

The comparison of the LIDAR-derived aerosol profiles, the balloon data and model simulations, allowed for the study/interpretation of the evolution of both the aerosol loads and the atmospheric MLH in this urban site. These aspects are presented and discussed hereafter.

\section{RESULTS}

\section{Analysis of the aerosol load}

An example of the LIDAR-derived aerosol extinction vertical profile is shown in Figure 1 (dashed line). This was collected on 20 July 2007 at about 8:00 UTC. LIDAR data were averaged over $1 \mathrm{~h}$ interval to optimise the signal-to-noise ratio. The relevant aerosol extinction as derived from the BBOPC size distribution data is also shown in Figure 1 for comparison (solid line). In this case, the computation was done using the Mie scattering theory (i.e. assuming the particles to be spherical) and a typical particle refractive index of $1.45-0.005 \mathrm{i}$.

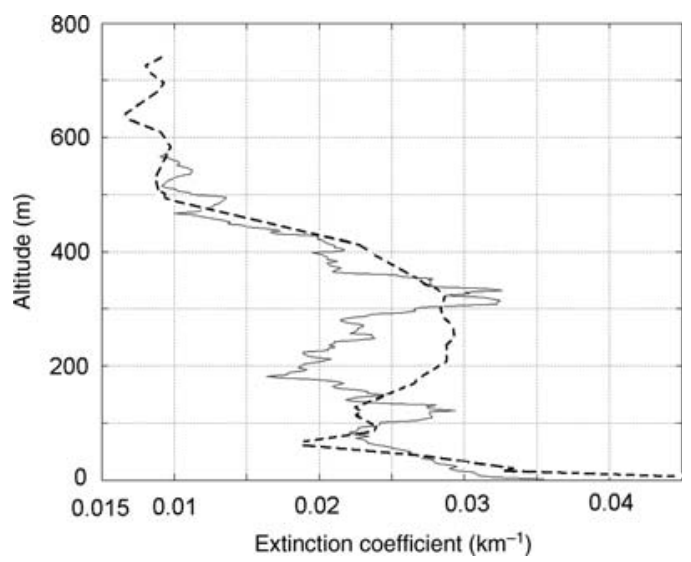

Figure 1. Aerosol extinction profile (at $855 \mathrm{~nm}$ ) as retrieved in Milan by LIDAR (dashed line) and derived from BB-OPC size distribution data (solid line). LIDAR data were averaged over $1 \mathrm{~h}$. Data refer to Grimm-Vaisala comparison 20 July 2007, 7:55 UTC. 
The agreement between the two data sets is rather good, considering that the BB-OPC data are collected at 2-m-vertical resolution, while LIDAR data are space averaged (running mean) over $\sim 50 \mathrm{~m}$. LIDAR data $<60 \mathrm{~m}$ have been extrapolated. It is worth highlighting the complex aerosol vertical structure observed.

\section{Analysis of the MLH}

The algorithm implemented for the determination of the mixing layer searches, over an hourly basis, the heights corresponding to local maxima of the logarithm of the range corrected signal (RCS or $S \times R^{2}$ ). To do so, the signal is firstly overlap-corrected and screened for clouds or haze by means of a threshold on the RCS. A running average over $50 \mathrm{~m}$ is performed to further increase the signal-to-noise ratio.

Due to the possible presence of a multiple-layer structure, this procedure may generate a certain number of points (identifying the main aerosol layers) in each of the 24 hourly bins. The MLH is then determined as the lowest height of the identified points. The ML height determined by this approach corresponds to the upper end of the first stratification above the LIDAR, usually coinciding with the top of the mixed layer (or bottom of the entrainment layer). Occasional stratifications occurring within the ML can be detected and rejected because of their short temporal duration with respect to ML evolution times.

A gradient method based on the direct numerical derivative of the particle number concentration along height is also adopted to evaluate MLH from BB-OPC observations. In particular, the MLH is chosen as the height at which the strongest gradient in particle concentration is found ${ }^{(15)}$. Finally, the MLH determination of the MM5 model is essentially based on the Holzworth (or 'air parcel') method $^{(16)}$. This considers the lowest height at which the virtual potential temperature exceeds the one at ground.

From the description above, it is evident that a problem on the definition of 'mixing layer' itself also

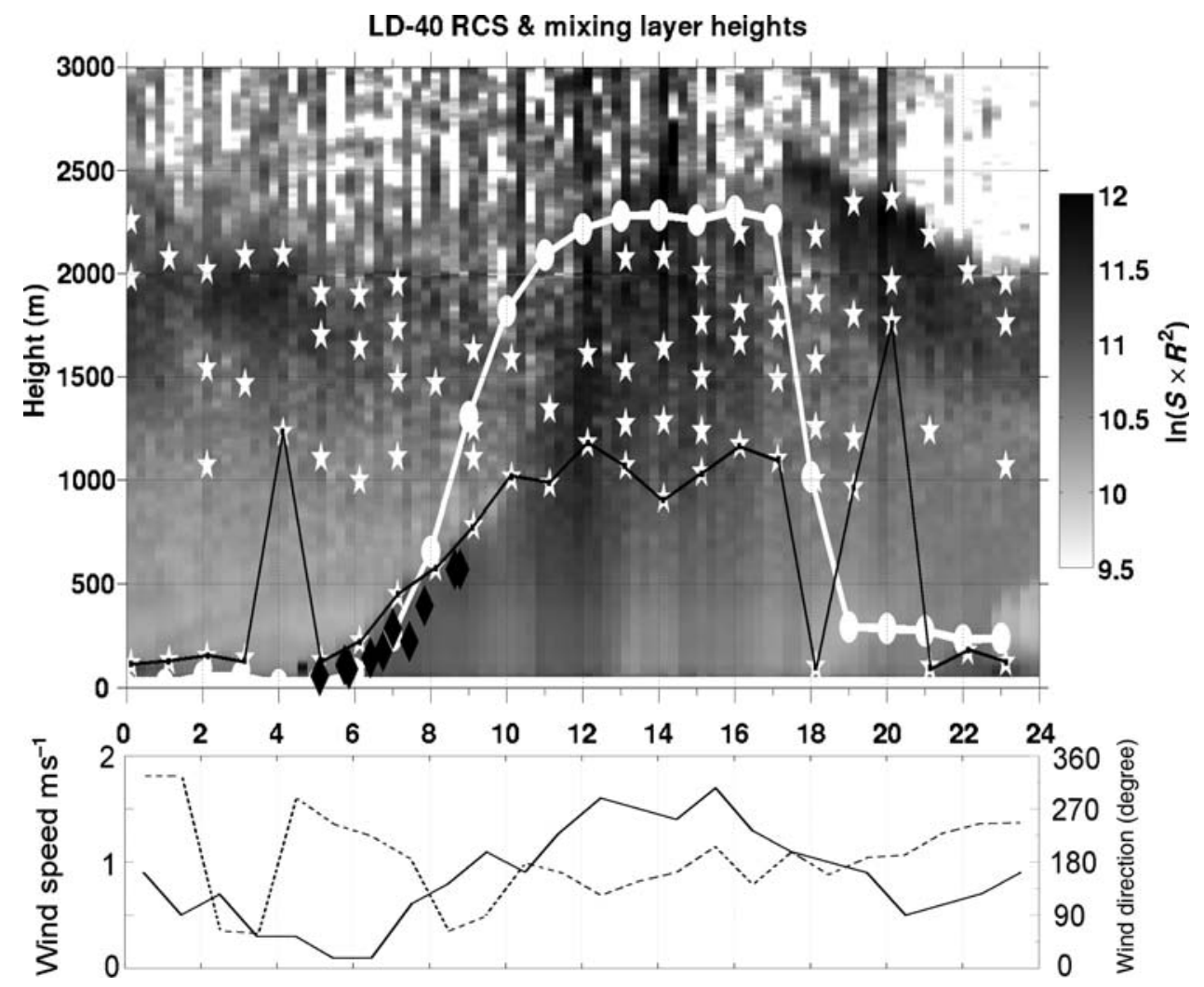

Figure 2. Typical summer case (16 July 2007) of aerosol distribution at Milan. Upper panel: LIDAR RCS (contour plot), relevant main aerosol layers (white stars) and MLH estimation (black line connected, white stars) compared with the MLH as derived from BB-OPC (black diamonds) and predicted by the MM5 model (white line connected, bullets); bottom panel: ground wind speed (full line) and direction (dashed line). 


\section{F. ANGELINI ET AL.}

exists: it is indeed well known that the comparison between these different quantities may lead to discordant results, depending on the conditions of the atmosphere $^{(13)}$.

Figure 2 (top panel) shows an example (16 July 2007) of the LIDAR daily RCS record (grey-scale contour plot).

A multiple-layer structure is clearly visible in the LIDAR trace, as also highlighted by the signal inflection points identified by the MLH algorithm (white stars). In particular, elevated aerosol layers (between 1000 and $2000 \mathrm{~m}$ ) are detected. These, typically observed in Summer, are caused by the diurnal convective uplift and constitute a 'residual layer' that is particularly evident at nighttime. Such layers can reside in the atmosphere for a long time if no (or too weak) particle removal (through precipitation or advection) occurs. The LIDAR-derived MLH is also shown in Figure 2 (black line connected-white stars). Starting from about 6 UTC, MLH evidently increases from nighttime values $(<100 \mathrm{~m})$ to a maximum of $\sim 1000 \mathrm{~m}$ at about 12 UTC. The LIDAR-derived MLH is then compared with the ones estimated by both the BB-OPC (black diamonds) and MM5 model (white line-connected bullets) in Figure 2. Finally, wind data are also reported (bottom panel) as useful in the overall interpretation. Figure 2 shows a rather good agreement between the LIDAR and BB-OPC MLH. Unfortunately, no BB-OPC data are available after 9 UTC. Conversely, model simulations evidently overestimate the MLH between 9 and 18 UTC. This might be due to the fact that the model calculates the MLH at the top of the 'entrainment layer' (which is 'seen' by the LIDAR as the 'turbulent' zone revealed by the presence of several aerosol layers, up to about $2000 \mathrm{~m}$, in the central part of the day). As mentioned, the LIDAR-derived MLH lays at the bottom of the entrainment layer.

A decrease in the aerosol content is observed in the early afternoon (after 12 UTC) in the ML. This coincides with the increase in wind speed due to the local breeze (Figure 2, bottom panel). Both the described 'entrainment zone effect' and the windinduced cleansing were often observed in the summertime LIDAR record. It should be also mentioned that MM5 systematically overestimated ground wind speeds, compared with the measured one. This may help explaining the higher MM5 MLH values through an overestimation of the model turbulent kinetic energy.

Significant differences between the observed and model-derived MLH were also found during the winter nighttime (not shown). In fact, during the night the MLH derived from LIDAR data is generally higher than the modelled one. However, it is important to notice that, under very stable conditions, the mixing layer can be lower than the lowest height the LIDAR can observe $(\sim 60 \mathrm{~m})$. This is a well-known problem ${ }^{(13)}$, and a major hindrance in retrieving the MLH by LIDAR. On the other hand, difficulties associated with the parameterisation of turbulence in the stable nocturnal planetary boundary layer also exist ${ }^{(17)}$. In fact, many PBL parameterisations simply set to a fixed value the MLH in such stable conditions.

Finally, it has to be mentioned that a collapse of the mixing layer after the sunset is hardly ever observed by the LIDAR. This is due to both misinterpretation of aerosol residual layers aloft and/or too low stratifications undetectable by the LIDAR (e.g. Figure 2).

\section{CONCLUSIONS}

LIDARs represent a powerful tool to sound the atmosphere and its constituents with rather high vertical and temporal resolution. Some examples of the ability of these systems to provide information on the aerosol vertical distribution and the atmospheric MLH were shown. The LIDAR ceilometer measurements employed in this study were extracted from a 1-year data set (January 2007 to February 2008) collected in the urban environment of Milan (Italy). A multiple-layer aerosol vertical structure (similar to those shown in Figures 1 and 2) was frequently observed. This questions the validity of the common assumption of an exponential-type aerosol vertical distribution $^{(18)}$.

A validation of the LIDAR aerosol extinction profiles and MLH estimates was performed using aerosol size distribution data from a BB-OPC. A good agreement was found between the aerosol extinction profiles derived by LIDAR and those computed from the BB-OPC data. Similarly, a good agreement was found between the MLH estimates from the two instruments, thus giving confidence on the capabilities of LIDAR ceilometers to provide trustworthy, continuous monitoring of the planetary boundary layer and MLH.

Conversely, the comparison of the observationsbased MLH values with those simulated by a mesoscale model (MM5) resulted to be more difficult due to both LIDAR instrumental limitations and model drawbacks (as difficulties at reproducing the meteorological field and/or parameterising the stable nocturnal boundary layer).

\section{ACKNOWLEDGEMENTS}

The authors would like to thank E. Bolzacchini and L. Ferrero (University of Milano 'Bicocca') for the BB-OPC data and relevant MLH analysis; P. Stocchi and G. Curci (CETEMPS) for the MM5 simulations, and ARPA Lombardia for the wind data. 


\section{FUNDING}

These studies have been conducted in the framework of the projects QUITSAT, funded by the Italian Space Agency, and AEROCLOUDS, funded by the Ministry of University and Research.

\section{REFERENCES}

1. IPCC (Intergovernmental Panel on Climate Change) Climate change 2007. The Physical Science BasisSummary for Policymakers. www.ipcc.ch/pdf/assessmentreport/ar4-wgl-spm.pdf (2007).

2. Al Saadi, J. et al. Improving national air quality forecasts with satellite aerosol observations. Bull. Am. Meteorol. Soc. 1249-1261 (2005). doi:10.1175/ BAMS-86-9-1249.

3. Arya, S. Pal: Air Pollution Meteorology and Dispersion (Oxford University Press) (1998).

4. Gobbi, G. P., Barnaba, F. and Ammannato, L. Estimating the impact of Saharan Dust on the PM10 record in Rome (Italy) in 2001. Atmos. Environ. 41, 261-275 (2007).

5. R. M. Measures: Laser Remote Sensing: Fundamentals and Applications (Krieger Publisher Company) (1992).

6. Klett, J. D. Stable analytical inversion for processing lidar returns. Appl. Opt. 20, 211-220 (1981).

7. Mitev, V. M., Grigorov, I. V. and Simeonov, V. B. Lidar measurements of atmospheric aerosol extinction profiles: a comparison between two techniques-Klett inversion and pure rotational Raman scattering methods. Appl. Opt. 31, 6469-6474 (1992).

8. Barnaba, F. and Gobbi, G. P. Lidar estimation of tropospheric aerosol extinction, surface area and volume: maritime and desert-dust cases. J. Geophys. Res. 106(D3), 3005-3018 (2001).
9. Melfi, S. H., Spinhirne, J. D., Chou, S.-H. and Palm, S. P. Lidar observations of vertically organized convection in the planetary boundary layer over the ocean. J. Clim. Appl. Meteorol. 24, 806-821 (1985).

10. Endlich, R. M., Ludwig, F. and Uthe, E. E. An automated method for determining the mixing layer depth from lidar observations. Atmos. Environ. 13, $1051-1056$ (1979).

11. Flamant, C., Pelon, J., Flamant, P. and Durand, P. Lidar determination of the entrainment zone thickness at the top of the unstable marine atmospheric boundary layer. Bound.-Lay. Meteorol. 83, 247-284 (1997).

12. Hennemuth, B. and Lammert, A. Determination of atmospheric boundary layer height from radiosonde and lidar backscatter. Boundary-Layer Meteorol. 120, 181-200 (2006).

13. Eresmaa, N., Karppinen, A., Joffre, S. M., Räsänen, J. and Talvitie, H. Mixing height determination by ceilometer. Atmos. Chem. Phys. 6, 1485-1493 (2006).

14. Troen, I. and Mart, L. A simple model of the atmospheric boundary layer; sensitivity to surface evaporation. Bound.-Lay. Meteorol. 37, 129-148 (1986).

15. Seibert, P, Beyrich, F., Gryning, S. E., Joffre, S., Rasmussen, A. and Tercier, P. Review and intercomparison of operational methods for the determination of the mixing height. Atmos. Environ. 34, 1001-1027 (2000).

16. Holzworth, G. C. Estimates of mean maximum mixing depths in the contiguous United States. Mon. Weather Rev. 92, 235-242 (1964).

17. Lee, S.-M., Giori, W., Princevac, M. and Fernando, H. J. S. Implementation of a stable PBL turbulence parameterization for the mesoscale model mm5: nocturnal flow in complex terrain. Bound.-Lay. Meteorol. 119(1), 109-134 (2006).

18. Kaufman, Y. J. and Fraser, R. S. Light extinction by aerosols during summer air pollution. J. Appl. Met. 22, 1694-1706 (1983). 